\title{
Adaptive Hierarchical Fuzzy controller for HVAC Systems in Low Energy Buildings
}

\author{
Basil Hamed*and Fadi Alami
}

Faculty of Engineering, Department of Electrical Engineering Islamic University of Gaza, Palestine

\begin{abstract}
Proper control for low energy buildings is more difficult than conventional buildings due to their complexity and sensitivity to operating conditions. In this paper, Adaptive Hierarchical Fuzzy control is used to control Heating, Ventilating and Air Conditioning (HVAC) System which is time varying nonlinear system. The proposed Controller is capable of maintaining comfort conditions under time varying thermal loads. Adaptive Hierarchical Fuzzy is consist of two levels; first fuzzy level is to control (Air temperature and Air quality); the second fuzzy level is to control the Error and Change of Error that comes from first level. A hierarchical structure is used to reduce the number of rules, trim redundant information and reduce the computing time required for the optimization. The controller is developed using a computer simulation of a virtual building contains most parameters of a real building. Fuzzy rules are learned from experts and system performance observations. Matlab program is used to simulate HVAC system and to see the results of the new controller.
\end{abstract}

Keywords: HVAC systems, Adaptive control, Hierarchical Fuzzy, Low Energy Buildings

\section{Introduction}

The consumption of energy by heating, ventilating, and air conditioning (HVAC) equipment in industrial and commercial buildings constitutes $40 \%$ of the world energy consumption [1]. In spite of the advancements made in microprocessor technology and its impact on the development of new control methodologies for HVAC systems aiming at improving their energy efficiency, the process of operating HVAC equipment in commercial and industrial buildings is still an inefficient and highenergy consumption process [2]. Classical HVAC control techniques such as the ON/OFF controllers (thermostats) and the proportional-integral-derivative (PID) controllers are still very popular because of their low cost. However, in the long run, these controllers are expensive because they operate at a very low-energy efficiency.

One important factor affecting the efficiency of air conditioning systems is the fact that most HVAC systems are set to operate at design thermal loads while actual thermal loads affecting the system are time-varying [3]. Therefore, control schemes that take into consideration time varying loads should be able to operate more efficiently and better keep comfort conditions than conventional control schemes. This paper presents Adaptive Hierarchical Fuzzy controller the HVAC system capable of maintaining comfort conditions within a thermal space with time varying thermal loads acting upon the system with high air quality. To achieve this objective, we carry out the design of an HVAC control system that counteracts the effect of thermal loads on the space comfort conditions. The controller achieves this objective by adapting the varying parameters of thermal loads acting upon the system and using the hierarchical fuzzy with two levels to take the appropriate control actions to maintain space comfort conditions.

First fuzzy level is adaptive level for varying parameters; it controls the deference temperature just after entering the room. The real temperature in the room and this varying in temperature due to slow spread nature of heating the air in an open large spaces [4]. It is due to changes happened in this space as large windows opened or any external disturbances. The second varying parameter is the quality of air inside this space cause, if place is crowded in this space $\mathrm{CO} 2$ concentration will change and the need of new air is essential, so a new cold air must enter the space. These varying parameters are nonlinear [5], and can't be expected when it will be

*Corresponding Author: Faculty of Engineering, Department of Electrical Engineering Islamic University of Gaza, Palestine, Electrical Engineering Department, Islamic University Gaza, Palestine, E-mail: bhamed@iugaza.edu 
changed so such an adaptive controller is needed, also an intelligent controller as Fuzzy control method is very useful and flexible with unknown systems.

\section{HVAC Systems and FLC}

In the specific case of HVAC systems, most works apply FLCs to solve simple problems such as thermal regulation, maintaining a temperature set point [6-8]. However, in this work combination of fuzzy control and adaptive scheme is used. First we use the principle or the structure of adaptive control to build the controller [9]. Then blocks that make the construction of adaptive control is fuzzy control Blocks. In this research we use Mamdani fuzzy control method [10]. The controller is consist of two fuzzy control levels with three inputs from temperature sensors and one output to heating system. Virtual model is used to study controller reactions.

\subsection{Heating, Ventilating, and Air Conditioning Systems}

An HVAC system is comprised by all the components of the appliance used to condition the interior air of a building. The HVAC system is needed to provide the occupants with a comfortable and productive working environment which satisfies their physiological needs. The system studied in this research is large buildings or large halls as: malls, hypermarkets, large study halls, indoor stadiums, factories, airports and so on.

The building that has been chosen is a virtual building model that presents one of the above systems. That it contains most of the parameters and specification of a large building and contains the actions that can be happened during system running. In a modern intelligent building, a sophisticated control system should provide excellent environmental control [11]. Figure 1 shows the building indoor space block and it is consisting of three main things: Inlet Air, Outlet Air and Sensors.

- Inlet Air: Inlet Air is the air enters the room or the space which required to be heated; inlet air may be recycled air or fresh air or mix between them, inlet air comes after Air Handling Unit (AHU).

- Outlet Air: Outlet air is the air which has been vacuumed from the room and returned to be recycled or to get rid of it.

- Temperature sensors: Temperature sensors are devices used to measure the temperature of a medium and there are many types of temperature sensors [12].

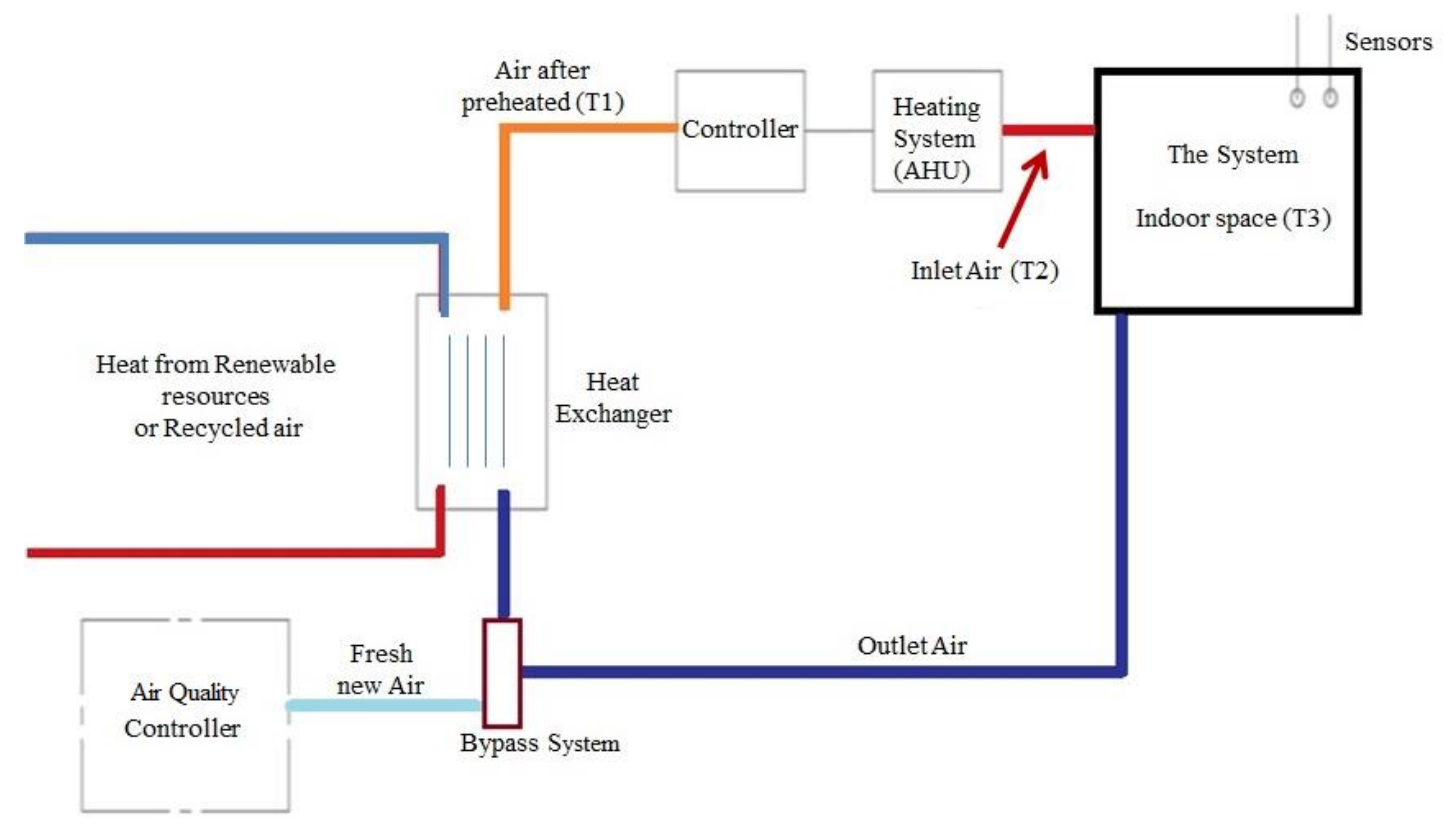

Figure 1: HVAC System 


\subsection{Adaptive hierarchical fuzzy controller}

In building automation, the objective of a global controller would be to maintain the indoor environment within the desired limits. In order to design a fuzzy system with a good amount of accuracy, an increase in the number of input variables to the fuzzy system results in an exponential increase in the number of rules required [13]. A hierarchical fuzzy logic control is used for accuracy, and to reduce the number of fuzzy rules. In addition, HVAC system is time variant nonlinear system. Then an adaptive controller must be used. Adaptive hierarchical fuzzy controller is used in this research as mention above to reduce the fuzzy rules and to take care of the time varying.

Adaptive Control is the type of control method used by a controller to adapt to a controlled system with parameters that vary, or are initially uncertain.

In large buildings as malls and hypermarkets and large centers it is extremely hard to establish mathematical model to the system due to the complexity of the system. A virtual system should be build and take varying parameters in consideration.
The essential varying parameters can as follows:

- Occupants Crowded and the amount of heat needed

- Air quality degradation and need of fresh air

- Doors and windows opening make disturbance of the system

It is hard to expect these varying parameters offline or online, and by applying the adaptive principle with fuzzy control. The proposed control system structure will be as shown in Figure 2.

System is consisting of:

1- System: Air temperature inside the room or hall.

2- Fuzzy Level1: Control the error of varying parameters to adjust main controller.

3- Fuzzy Level2: Is the main temperature controller.

4- Disturbances: As opining windows or doors and Co2 concentration.

5- Temperature T0: The reference Air Temperature.

6- Temperature T1: Air Temperature after disturbances.

7- Temperature T2: Air Temperature after being heated from heating system (AHU).

8- Temperature T3: Air Temperature inside the room. 9- Desired performance: The rules of fuzzy controllers from system experts.

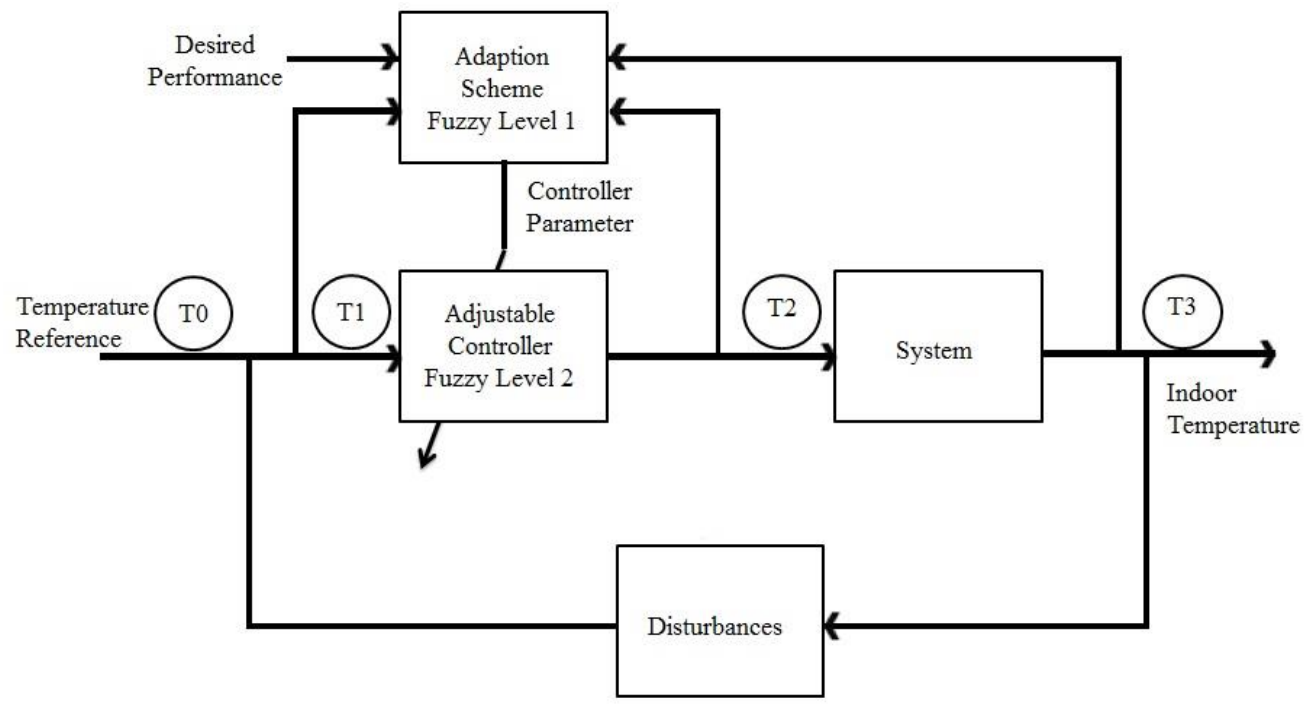

Figure 2 Controller Scheme

\subsection{Controller working methods}

The HVAC system is time varying and an intelligent controller is applied as fuzzy control approach. In order to design an intelligent controller an increase in the number of input variables and input values (sets) to the fuzzy system results in an exponential increase in the number of rules required. If there are $\mathbf{n}$ input variables and $\mathbf{m}$ fuzzy sets are defined for each of these, then the number of rules in the fuzzy system is $\mathbf{m}^{\mathbf{n}}$. The proposed system consist of 3 inputs and 5 fuzzy sets then $5^{3}=125$ rules, so we can imagine the rules complexity if the number of inputs or sets increased. So we need to use hierarchical fuzzy; the number of rules will be decreased from 125 to 50 . This can be explained as: we have two fuzzy levels 
each fuzzy level has 2 inputs and 5 fuzzy sets so the number of rules will become $5^{2}+5^{2}=50$. The control method is depending on two control level; in level 2 Fuzzy inputs are the error and the result of fuzzy control. Level 1(Change of Error), and Error is deference between reference air temperature (T0) and the air temperature inside the room (T3) - as any traditional close loop control - and fuzzy control level 1(Change of Error) correct control operation of fuzzy level 2 because of these reasons:

1- When a new fresh air enters the system there is no feedback for this change and based on that no change in the error $\mathrm{T} 0-\mathrm{T} 3$.

2- The nature of heat spread is slow, so it can't know the value of air temperature. Just after heating the system and if doesn't take it in consideration, it will never mind that heating system works as On/Off method (Open loop control for heating system).

These problems make fuzzy control level 1 is very important to correct this control loop. Fuzzy control
Level 1 adapt to the varying parameters if it comes from disturbances or unexpected system performance. This control method depends on compensating the loss of air temperature by the same amount of loss. In other words, if the reference air temperature (T0) was $30 \mathrm{C}^{\circ}$ and room temperature (T3) was $20 \mathrm{C}^{\circ}$ then controller output must be $40 \mathrm{C}^{\circ}$. As we see there is 10 $\mathrm{C}^{\mathrm{o}}$ losses the controller compensate it with $10 \mathrm{C}^{\circ}$ plus the reference point and this method is to reach the stability desired point as fast as we can, but we use some limits to upper heat air temperature of controller output (T2). The amount of compensation is programed with fuzzy control rules or by fuzzy output membership. Inputs of Fuzzy controller level 1 will be:

A. (T0-T1) that observe the variations come from entering a fresh new air from Air Quality Performance.

B. $(\mathrm{T} 2-\mathrm{T} 3)$ that observe the deference between air temperature enters the room (after heating system) and air temperature inside the room. System and control structure is shown in Figure 3.

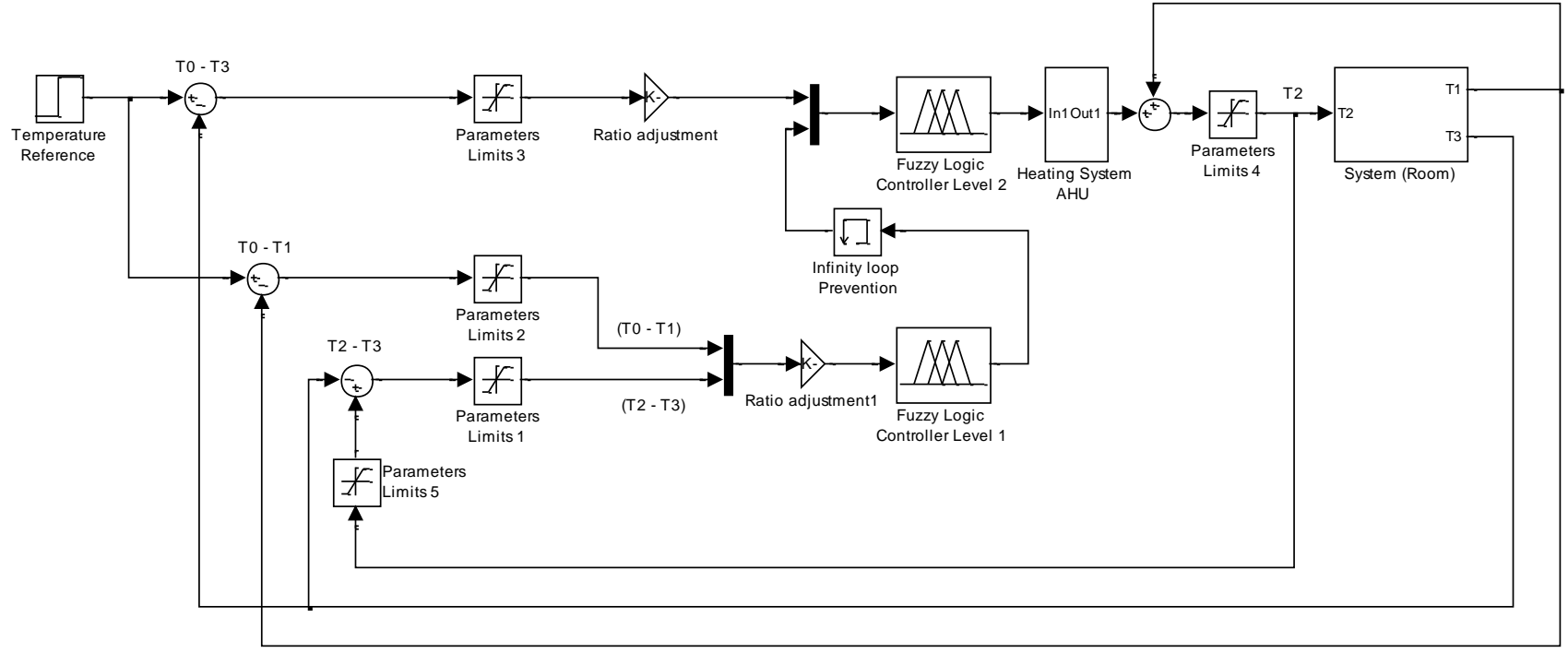

Figure 3. HVAC Controller Simulink Block Diagram

\section{Results}

Results of Adaptive hierarchical fuzzy controller method with a desired reference temperature of $30 \mathrm{C}^{\circ}$ is shown in Figure 4. Figure 4 shows at time:

- $\mathrm{T}=0$ (system initial value): If $\mathrm{T} 1$ and $\mathrm{T} 3$ is -20 controller will work to maximum and maximum is 30 degrees upper the air temperature enters heating system.

- $\mathrm{T}=2$ : If $\mathrm{T} 1$ and $\mathrm{T} 3$ are 20 and the desired is 30 then compensation control will give 40 or higher but upper limit specification is 40 and this is upon building management requirements.

- T=4: If T3 reached the required value or reference value and $\mathrm{T} 1$ is 10 degrees then the controller will compensate lose in temperature only for fresh new air to 30 degrees not higher because the Error $=0$.

- T=6: If system reach desired temperature the controller will stop heating air $\mathrm{T} 0=\mathrm{T} 3=\mathrm{T} 2$ 


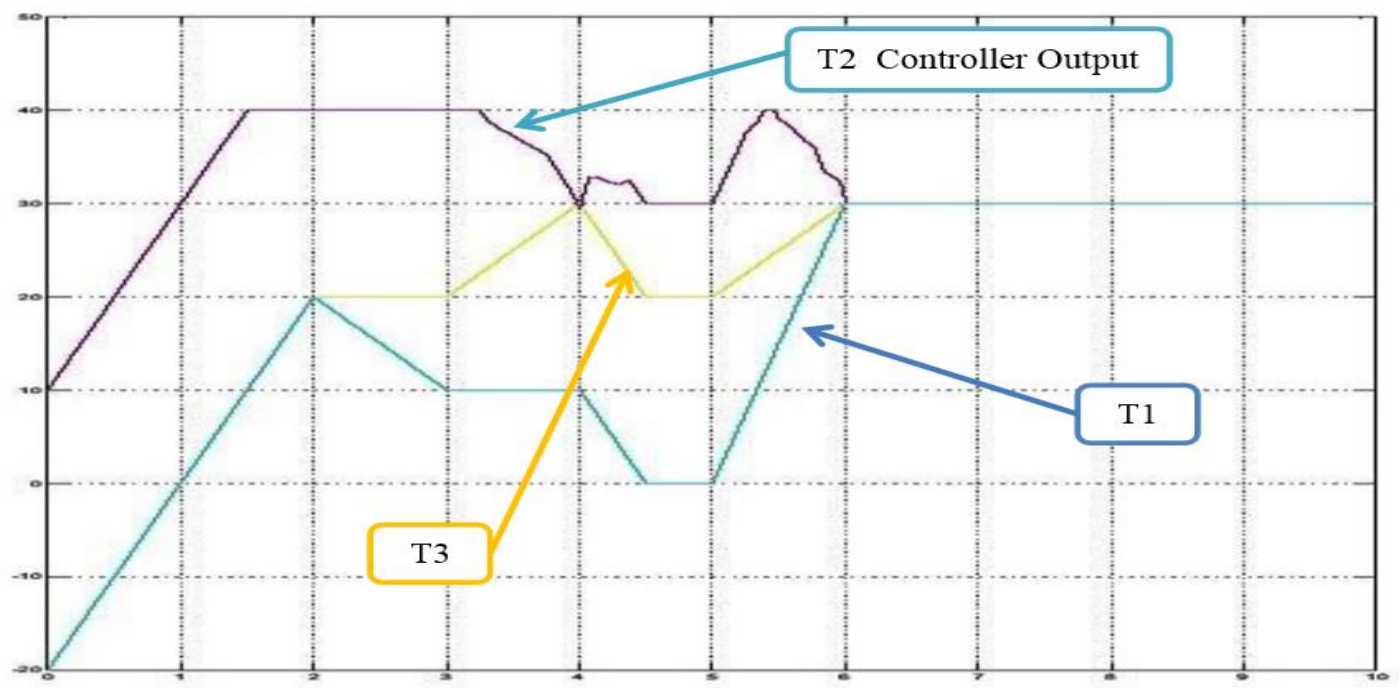

Figure 4. Compensation Method Result: Pink Line is T2, Yellow Line is T3 and Blue Line is T1

\subsection{Fuzzy Inference System (FIS)}

In general, the inference is a process to obtain new information by using existing knowledge. The fuzzy inference engine performs the actual decision-making process [14]. The fuzzy rule base consists of a collection of fuzzy IF-THEN rules. In this paper, the rules are summarized in Table 1.

The rules are stated as follows [15]: $\mathrm{R}(\mathrm{k}):$ IF $x_{l}$ is $A_{l k}$ and $x_{2}$ is $A_{2 k}$ THEN y is $Q_{k}$ for $k=1,2, \ldots, n$

where $x_{1}, x_{2} \in U$, and $y \in R$ are the input and output of the fuzzy logic system, respectively, $A_{l k}, A_{2 k}$, and $Q_{k}$ are labels of fuzzy sets $U_{1}, U_{2}$, and $R$ representing the $k^{\text {th }}$ antecedent pairs and consequent pair respectively and $\mathrm{n}$ is the rules number of rules. For instance, IF $x_{l}$ is SMALL and $x_{2}$ is MEDIUM THEN $y$ is LARGE (see Table 1).

Rules can be presented from Matlab as rule surface as shown in Figure 5.

Table 1. Fuzzy Associative Memory

\begin{tabular}{|c|c|c|c|c|c|}
\hline $\begin{array}{c}\text { Input 1/ } \\
\text { Input 2 }\end{array}$ & Zero & Small & Medium & Large & $\begin{array}{c}\text { Very } \\
\text { Large }\end{array}$ \\
\hline Zero & $\mathrm{Z}$ & $\mathrm{S}$ & $\mathrm{M}$ & $\mathrm{L}$ & $\mathrm{VL}$ \\
\hline Small & $\mathrm{S}$ & $\mathrm{M}$ & $\mathrm{L}$ & $\mathrm{VL}$ & $\mathrm{VL}$ \\
\hline Medium & $\mathrm{M}$ & $\mathrm{L}$ & $\mathrm{VL}$ & $\mathrm{VL}$ & $\mathrm{VL}$ \\
\hline Large & $\mathrm{L}$ & $\mathrm{VL}$ & $\mathrm{VL}$ & $\mathrm{VL}$ & $\mathrm{VL}$ \\
\hline $\begin{array}{c}\text { Very } \\
\text { Large }\end{array}$ & $\mathrm{VL}$ & $\mathrm{VL}$ & $\mathrm{VL}$ & $\mathrm{VL}$ & $\mathrm{VL}$ \\
\hline
\end{tabular}

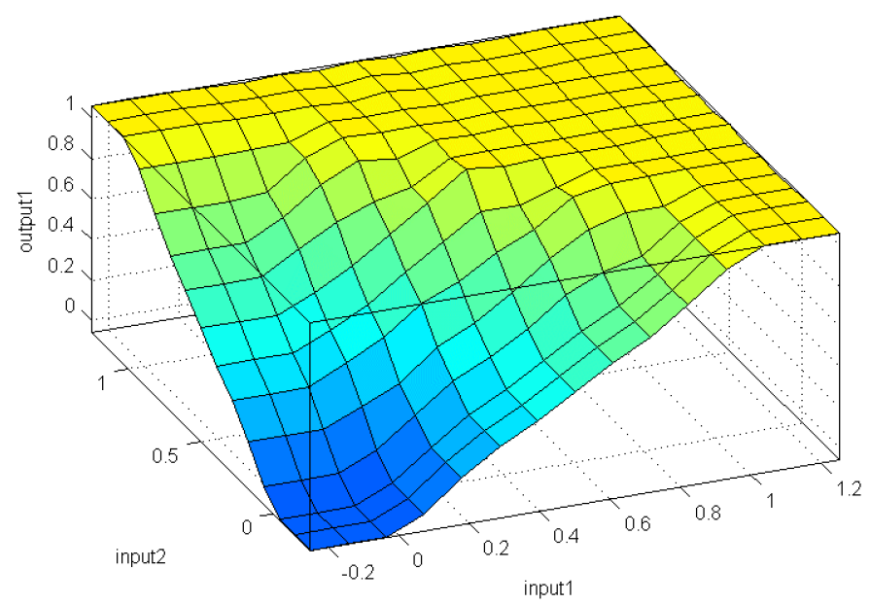

Figure 5. Fuzzy Rule Surface

\subsection{Matlab functions and simulation:}

We use saturation block to give boundaries for the values enter controllers or the output of these controllers. As an example let us consider the Error value is (T0 - T3) and if we suppose the initial value of the system $\mathrm{T} 0=30$ and $\mathrm{T} 3=-20$ thus $\mathrm{T} 0-\mathrm{T} 3=30$ $+20=50$, if $\mathrm{T} 0=30$ and $\mathrm{T} 3$ is over heated and equal 40 then $\mathrm{T} 0-\mathrm{T} 3=30-40=-10$. But the variation deference boundaries is between 0 and 30 lower than 0 will be 0 and the controller will stop heating air and higher than 30 will be 30 and the controller will work at maximum. Gain block is used to correct the ratio of the boundaries of the system. If the system works between 0 and 30 degrees the controller works in unity from 0 to 1 , then the correction factor will be 30. If the system works from 0 to 40 then the correction factor will be 40 . 
Memory block is used because the control loop is hierarchical fuzzy close loop. That means fuzzy controller level 1 is the input of fuzzy controller level 2 , then the output of fuzzy controller level 2 will return to fuzzy controller level 1 as shown in Figure 6. So the memory block is used to prevent an infinity loop for Matlab processes by a not effective delay between two controllers.

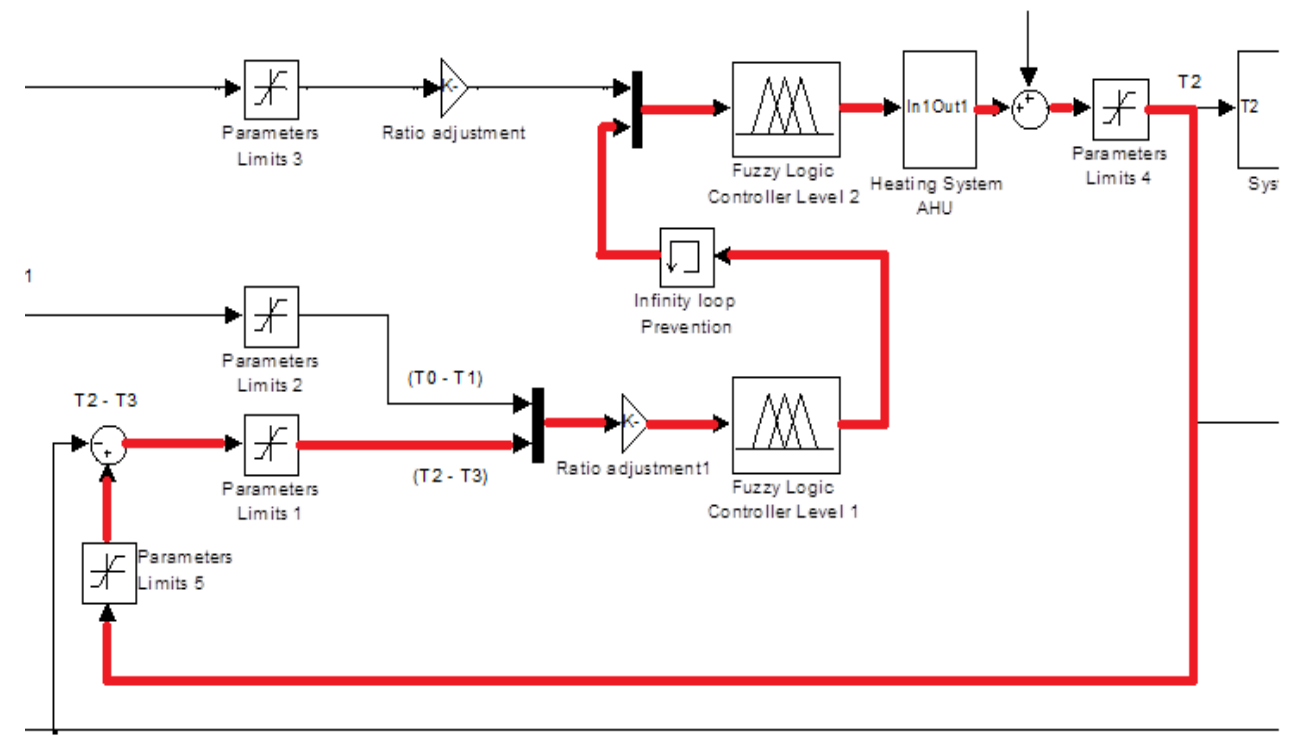

Figure 6. Infinity loop problem

\section{Conclusion}

Adaptive hierarchical fuzzy control approach is introduced in this paper to control a HVAC system for Low energy buildings. This novel approach reduces the fuzzy rule numbers but still maintains the linguistic meaning of fuzzy variables and adapt to changes and disturbances that may be happened to the system in any time. The proposed control method seeks to reach the stability point as fast as possible so consume less energy and reach the desired temperature as fast as possible. This method gives good results; compared to previous researches working on time varying nonlinear HVAC systems, it is special due to their flexibility and adaptively to any system model. Hierarchical fuzzy method helps to reduce the number of rules used in this controller and makes it easy to understand rules evaluation and make it possible to increase the number of inputs without fearful of rules increase. Hierarchical fuzzy make it easy to partition the controller and gives a better understand of controller running. Adaptive Hierarchical Fuzzy Controller also preserve Air Quality beside Air temperature to give overall good comfort level for occupants. The controller pre-heat the new air that enter the system and heated it to keep indoor air temperature not changed and this thing help the system to stay stable. The results of this work should be ready for implementation in real buildings for the specific studied systems. This methodology could then be applied to other systems and progressively implemented at industrial level

\section{References}

[1] Clas A. Jacobson, "Energy Efficient Buildings A Systems Approach Market Status", Regulatory Pressure, Technology Gaps Stanford Energy Seminar May 9, 2011

[2] Farinaz Behrooz1, Abdul Rahman Ramli and Khairulmizam Samsudin "A survey on applying different control methods approach in building automation systems to obtain more energy efficiency", International Journal of the Physical Sciences Vol. 6(9), pp. 2308-2314, 4 May, 2011

[3] John Lataire, Frequency Domain Measurement and Identification of Linear, Time-Varying Systems.

[4] Sadik Kakaç and Hongtan Liu (2002). Heat Exchangers: Selection, Rating and Thermal Design (2nd ed.). CRC Press. ISBN 0-8493-0902-6

[5] Hassan K. Khalil, Nonlinear Systems Third Edition, ISBN 0-13-067389-7, Prentice Hall 2002

[6] M. Arima, E.H. Hara, and J.D. Katzberg, "A fuzzy logic and rough sets controller for HVAC systems," in Proc. of the IEEE WESCANEX'95, vol. 1, NY, 1995, pp. 133-138. 
[7] P.Y. Glorennec, "Application of fuzzy control for building energy management," in Building Simulation: International Building Performance Simulation Association 1, Sophia Antipolis: France, 1991, pp. 197-201.

[8] S. Huang, and R.M. Nelson, "Rule development and adjustment strategies of a fuzzy logic controller for an HVAC system - Parts I and II (analysis and experiment)," ASHRAE Transactions, vol. 100, no. 1, pp. 841-850, 851-856, 1994.

[9] Astrom, Karl Adaptive Control. Dover,2008

[10] E. H. Mamdani and S. Assilian, "An experiment with in linguistic synthesis with a fuzzy logic controller," International journal of Man-Machine studies, vol. 7, pp. 1-13, 1975.

[11] M. Arima, E.H. Hara, and J.D. Katzberg, "A fuzzy logic and rough sets controller for HVAC systems," in Proc. of the IEEE WESCANEX'95, vol. 1, NY, 1995, pp. 133-138.
[12] Ardemis Boghossian, James Brown, Sara Zak,Temperature Sensors, 2006 Online: https://controls.engin.umich.edu/wiki/index.php/Tem peratureSensors

[13] Timothy J. Ross, Fuzzy Logic with Engineering Applications, Third Edition (C) 2010 John Wiley \& Sons, Ltd. ISBN: 978-0-470-74376-8

[14] Mohammed S. EL-Moghany, Sun and Maximum Power Point Tracking in Solar Array Systems Using Fuzzy Controllers Via FPGA, Islamic University,2011

[15] Riza,B. Sheldon,T., Fuzzy Systems Design Principles Building Fuzzy IF-THEN Rule Bases, IEEE PRESS(1997). 